\title{
Coordinated Shirking
}

\author{
Nicholas H. Tenev*
}

November 26, 2018

\begin{abstract}
In the financial crisis of 2008, losses on popular new securitized products far exceeded predictions. This paper studies this episode with a model of technology adoption: a principal tries to induce costly effort from a group of agents charged with vetting new technology. The principal is unwilling to simultaneously punish large groups of agents, so they shirk when coordination is possible. Widely applicable technology expands productive possibilities but also provides an opportunity for coordinated shirking, and can thus lead to widespread production failure. Furthermore, even agents who learn that they are using flawed technology may continue to do so.
\end{abstract}

JEL codes: D21; J33; O32.

Keywords: Principal-agent; financial crisis; technological innovation.

\footnotetext{
*Office of the Comptroller of the Currency, 400 7th St. SW, Washington DC 20219 . Email: nicholas.tenev@occ.treas.gov. The views expressed in this paper are those of the author and do not necessarily reflect those of the OCC or the US Department of the Treasury. I am grateful to Enghin Atalay, Shane Auerbach, Alexander Clark, Dean Corbae, Steven Durlauf, Chao Fu, and Rasmus Lentz for helpful comments and suggestions.
} 


\section{Introduction}

The financial crisis of 2008 was preceded by a credit boom, which was facilitated by advances in financial technology. Gaussian copula models in particular were developed in the late 1950's but became popular with practioners in the early 2000's, and were ubiquitous in pricing collateralized debt obligation (CDO) including securities backed by subprime mortgages. ${ }^{1}$ This technology became public knowledge in the finance community (MacKenzie and Spears $\left.(2014)^{2}\right)$ and allowed risky loans to be bundled into seemingly safer securities, making them palatable to investors with less appetite for risk. But when more borrowers than predicted defaulted at once, the securitized products formed from their loans lost much of their value.

A large asset price shock does not necessarily imply mistakes in modeling. However, Zimmer (2012) finds that the degree to which house prices are related is insufficiently captured by a Gaussian copula, even using data available before 2002. And MacKenzie and Spears (2014) find that industry practioners expressed "pervasive dissatisfaction" with Gaussian copula models even as early as 2006. So despite uncertainty and even pessimism about the use of a new financial technology, its use continued. ${ }^{3}$ But why?

This paper provides an answer to this puzzle, modeling an agency friction that can cause a particularly useful innovation to precipitate a downturn in aggregate production. Specifically, workers tasked with evaluating new technology may shirk their duty when a technology is sufficiently widespread in use, since their employers may not credibly be able to threaten to fire all of them. The model is simple, and cannot capture all the subtlety of model selection and evaluation, let alone the myriad other factors contributing to the financial crisis. Nonetheless, it cleanly illuminates an important tension: a

\footnotetext{
${ }^{1}$ Coval et al. (2009) provide a primer on securitized products and their role in the crisis; see also Zimmer (2012).

${ }^{2}$ MacKenzie and Spears (2014) present a fascinating account of the use of Gaussian copula models and their role in the crisis, featuring selections from 95 interviews of financial services workers of which 29 took place before July 2007.

${ }^{3}$ In fact, MacKenzie and Spears (2014) find that traders who wanted to use models other than Gaussian copulas had trouble convincing risk controllers that their positions were properly hedged. Thus while using the new technology was essentially free, not using it was made even more difficult by risk policies than this paper assumes.
} 
technology's popularity can belie its unsoundness.

In the model, a lone principal seeks to induce effort from a group of agents. New technology arrives exogenously each period; using it usually increases agents' production but on rare occasions causes production to fail. Those agents with access to the new technology can exert costly research effort to obtain a noisy signal of its promise. After obtaining this signal (or shirking and seeing no signal), these agents decide whether or not to use the new technology. The principal is unable to observe the agents' effort or the signal, but she can observe their production. She is able to punish agents who fail to produce, but at a cost which is convex in the number of agents punished. The main idea is that the principal may be unable to credibly threaten punishment to large groups of agents who shirk simultaneously: a "too many to fail" problem. This is similar to the work of Acharya and Yorulmazer (2008, 2007) and Farhi and Tirole (2012), in which the promise of government bailouts in an aggregate downturn can prompt banks to correlate the returns of their investments. However, the focus here is on the friction introduced by the interaction of technological progress with the convexity in the principal's cost of punishment.

In the model, even workers who find out that the technology is flawed may continue to use it, since they do not fear punishment for doing so. This behavior has a flavor of herding; Devenow and Welch (1996) summarize papers on herding in financial markets. Others (e.g. Acharya and Richardson (2009) and Rajan (2008)) have noted that analysts' compensation structures gave little incentive to care about the long-term performance of their work (a theme repeated here), but do not explain why reputation concerns or the threat of firing were not more effective restraints. This paper specifically explains why the threat of firing workers for poor performance may have been inadequate in these cases. Furthermore, it explicitly links a production downturn to the advent of a new technology, potentially helping to explain the timing of the financial crisis.

It also illuminates several possible solutions to the threat of coordinated shirking. First, expectations are important: if workers for some reason expect that nobody else is shirking, they too will 
be diligent. Second, shifting worker compensation from variable (bonuses) to fixed (salary) may exacerbate shirking, as it uncouples workers' incentives from their output. Finally, the problem of coordinated shirking can be resolved if there is a commonly known complete strict ordering of workers (such as seniority) that the firm can use as a basis for punishment. The most senior person of any group of prospective shirkers will be motivated to exert effort, unraveling the shirking behavior of the group.

Section 2 introduces the model. Section 3 derives conditions for equilibria, calculates production loss compared to the first-best case, and gives an example to aid intuition. Finally, Section 4 applies the model to the financial crisis and discusses policy implications. The Appendix considers another application of paper's model: herding in crime, such as speeding or looting, where the presence of other criminals encourages criminal activity by reducing the chance of punishment. This case has been considered by e.g. DiPasquale and Glaeser (1998); Becker (1968) is the foundational analysis of the economics of crime. This paper's relative contribution is to highlight the significance of agent anonymity: if there exists a natural ordering of agents, the principal can punish according to that ordering, causing the coordinated shirking to unravel.

\section{Model}

Time is discrete and infinite: $t=0,1,2, \ldots$. There exists a continuum of risk-neutral agents of measure one, ${ }^{4}$ indexed by $i \in[0,1]$, and one risk-neutral principal. The agent maximizes expected wages and the principal maximizes expected output less wages; they share a common time discount rate $\delta \in(0,1)$.

\footnotetext{
${ }^{4}$ Using a continuum of agents rather than a finite set of discrete agents eases the analysis, but is not qualitatively important.
} 


\subsection{Individual production}

An individual agent $i$ 's production function is

$$
f_{i t}=\left(1-\tau_{i t} \mu_{i t}\left(1-\chi_{t}\right)\right) a_{i t}
$$

The arguments are individual $i$ 's technology level $a_{i t}$, technology choice $\tau_{i t} \in\{0,1\}$, and an indicator of whether or not the new technology is sound $\chi_{t} \in\{0,1\}$. Agent $i$ produces his technology level $a_{i t}$ unless he has access to a new technology $\left(\mu_{i t}=1\right)$, chooses to use it $\left(\tau_{i t}=1\right)$, and it turns out to be unsound $\left(\chi_{t}=0\right)$. The principal owns the output of production.

\subsection{Technology}

Each period, new technology arrives exogenously. It affects the production of a fraction $\mu_{t}$ of the unit mass of workers, where $\mu_{t}$ is a random variable with support on the unit interval.

Specifically, assume that each period $\mu_{t}$ is drawn from a distribution $Q$, independent of history:

$$
\mathrm{P}\left[\mu_{t}<\mu\right]=Q(\mu)
$$

For agent $i$ let $\mu_{i t}$ be a binary variable indicating whether or not period $t$ 's new technology is applicable to agent $i$. The chance that the technology is relevant is $\mu_{t}$ :

$$
\begin{gathered}
\mathrm{P}\left[\mu_{i t}=1\right]=\mu_{t} \\
\mathrm{P}\left[\mu_{i t}=0\right]=1-\mu_{t} .
\end{gathered}
$$

The agents for whom the new technology is relevant are those for whom $\mu_{i t}=1$ and these chances 
are independent across agents, so

$$
\int_{0}^{1} \mu_{i t} d i=\mu_{t}
$$

The individual's technology level is $a_{i t}$, which indicates potential production. Technology is given by

$$
a_{i t}=1+\tau_{i t} \mu_{i t} g
$$

Here $\tau_{i t}$ is an indicator variable which is 1 if agent $i$ chooses to use the new technology and 0 otherwise. The new technology expands productivity by $g>0$ percent, but is only available to agent $i$ if $\mu_{i t}=1$, which occurs with probability $\mu_{t}$.

The new technology is sound with probability $\pi$, indicated by the binary random variable $\chi_{t}$ :

$$
\begin{gathered}
\mathrm{P}\left(\chi_{t}=1\right)=\pi \\
\mathrm{P}\left(\chi_{t}=0\right)=1-\pi .
\end{gathered}
$$

The random variables $\left\{\chi_{t}\right\}$ are independent across time.

\subsection{Effort/shirking}

When given the opportunity to use a new technology $\left(\mu_{i t}=1\right)$, agents cannot directly observe if it is sound $\left(\chi_{t}\right)$. However, they have the opportunity to undertake costly effort (E) to obtain a noisy signal

of $\chi_{t}$. Specifically, by paying a cost $c$, the agent learns the value of $\eta_{t}$, where $\varepsilon \in\left[0, \frac{1}{2}\right]$ is the chance that the signal is wrong:

$$
\begin{gathered}
\mathrm{P}\left(\eta_{t}=\chi_{t}\right)=1-\varepsilon \\
\mathrm{P}\left(\eta_{t}=1-\chi_{t}\right)=\varepsilon .
\end{gathered}
$$


The random variables $\left\{\eta_{t}\right\}$ are independent across time. Forgoing effort will, as is usual in the literature, be called 'shirking' (S).

\subsection{The principal}

The principal pays each agent $w a_{i t}$ before production is realized. The principal then receives the fruits of production: $\left\{f_{i t}\right\}_{i \in[0,1]}$, such that aggregate production is given by

$$
f_{t}=\int_{0}^{1} f_{i t} \mathrm{~d} i
$$

The principal cannot observe each agent's effort, but can observe output, and decides whom to punish. The cost of punishing a fraction $\lambda$ of the agents is $r(\lambda)$. This cost is assumed to be weakly increasing in the number of agents punished and convex. Also assume that there are negligible fixed costs to punishing agents ${ }^{5}$, so $r(0)=0$. Punished agents exit the game and receive the outside option $b$; they are replaced by agents identical to them.

Convexity of the punishment is a crucial assumption in the model. In the worker/firm context, punishment is akin to firing and replacing a worker. There may be a small number of people who are nearby and qualified for the job whom the firm can use to replace a small number of workers at low cost. Replacing large numbers of workers, though, may oblige the firm to pay relocation and/or retraining costs for some of the new hires.

The principal does not have to punish all agents who fail to produce, but rather can punish failure at a rate $\gamma$, which may depend on the number of potential failures $\mu$, such that an agent whose production fails is punished with probability $\gamma(\mu)$.

\subsection{Timing}

Each period, the following occur:

\footnotetext{
${ }^{5}$ If there are fixed costs, the principal may prefer not to punish small numbers of failures.
} 
1. A technology state $\mu_{t}$ is realized

2. Agents with access to the new technology $\left(\mu_{i t}=1\right)$ choose whether to exert research effort (E) or to shirk $(\mathrm{S})$

3. Agents who chose effort pay a cost $c$ and receive a signal $\eta_{t}$ of the new technology's promise, $\chi_{t}$

4. Agents with access to the new technology choose whether or not to use it $\left(\tau_{i t}\right)$

5. Agents receive $w a_{i t}$

6. Production $f_{i t}$ is realized for each agent, given technology $a_{i t}$ and the realization of $\chi_{t}$

7. The principal decides what fractions of agents to punish (at a cost $r(\lambda)$ of replacing a fraction $\lambda$ of the agents)

8. Punished agents receive $b$ and exit the game; the rest continue to the next period

In the worker-firm application, this is equivalent to workers negotiating a salary equal to a fraction $w$ of their prospective productivity, which they are paid unless they are fired. Using the same financial technology made it easier for traders to claim the full expected future profits of such deals as justification for (current) bonuses, since the deals were priced using standard formulae (MacKenzie and Spears (2014)). This corresponds well to the timing of the model, in which workers receive payment for prospective output before output is actually realized. The case of compensation based on performance is discussed in 4.1 .

\subsection{Strategies}

Each agent with access to a new technology must choose effort (E) or shirking (S), which may depend on how widely useful the technology is $\left(\mu_{t}\right)$. For each realization of signal $\eta_{t}$ as well as a null signal 
(if the agent shirks), the agent must choose whether to use the new technology. So an agent's strategy is a map from $[0,1]$ (the support of $\mu_{t}$ ) to $\{\mathrm{E}, \mathrm{S}\} \times\{0,1\}^{3}$.

Given that a fraction $\bar{f}$ of agents produced successfully, the principal's strategy consists of a rate $\gamma(\bar{f}):[0,1] \rightarrow[0,1]$ which determines the fraction of agents whose production failed to punish. If all agents pursue the same strategy, this is equivalent to writing $\gamma(\mu)$, since either $\mu_{t}$ agents will fail or none will.

\section{Results}

\subsection{First-best}

Fix a new technology $\mu_{t}$ and focus on the problem of an agent who has access to it. By ignoring the new technology, the agent is guaranteed production of 1 .

The expected production if the agent does not exert research effort (shirks) but uses the new technology is

$$
\mathrm{E}\left(f_{i t} \mid e_{i t}=0, \tau_{i t}=1\right)=\pi(1+g) .
$$

If the agent does exert research effort and receives a favorable signal $\left(\eta_{i t}=1\right)$, expected production using the new technology is

$$
\mathrm{E}\left(f_{i t} \mid e_{i t}=1, \eta_{t}=0, \tau_{i t}=1,\right)=(1-\varepsilon)(1+g) .
$$

This is greater than the production of one guaranteed by choosing $\tau_{i t}=0$ if

$$
g>\frac{\varepsilon}{1-\varepsilon}
$$

If the agent exerts research effort and receives an unfavorable signal $\left(\eta_{i t}=0\right)$, expected production 
using the new technology is

$$
\mathrm{E}\left(f_{i t} \mid e_{i t}=1, \eta_{t}=1, \tau_{i t}=1,\right)=\varepsilon(1+g) .
$$

Trusting the signal and forgoing the new technology will be preferable if

$$
g<\frac{1-\varepsilon}{\varepsilon} .
$$

Thus it will be efficient for agents who exert effort to act according to the signal received if

$$
g \in\left(\frac{\varepsilon}{1-\varepsilon}, \frac{1-\varepsilon}{\varepsilon}\right) .
$$

Expected production when exerting effort is then

$$
\mathrm{E}\left(f_{i t} \mid e_{i t}=1\right)=\pi \varepsilon+(1-\pi)(1-\varepsilon)+\pi(1-\varepsilon)(1+g) .
$$

This means that exerting effort is efficient if the cost $(c)$ and chance the signal is wrong $(\varepsilon)$ are small enough relative to the chance of the technology being good $(\pi)$ and the amount it improves production, $(g)$ :

$$
c<\pi(1-\varepsilon) g-(1-\pi) \varepsilon .
$$

For the remainder of the paper, assume that 2 and 3 hold. This is the basic requirement to ensure an interesting problem. If all agents were exerting effort, any failures in production would owe to chance. Since punishing agents is costly, in the first-best case all agents would exert effort (since it is efficient) and the principal would never punish. Given technology $\mu_{t}$, expected aggregate production in the first-best case is

$$
\int_{0}^{1} f_{i t} \mathrm{~d} i=\left(1-\mu_{t}\right)+\mu_{t}[(1+\pi g)(1-\varepsilon)+\pi \varepsilon]
$$


Total welfare is simply output less agent effort costs, since wages are a transfer. Total welfare in the first-best case is

$$
\left(1-\mu_{t}\right)+\mu_{t}[(1+\pi g)(1-\varepsilon)+\pi \varepsilon-c]
$$

\subsection{Equilibria}

As in other principal-agent settings, it is a Nash equilibrium here for agents to always shirk and for the principal to never punish. However, the more interesting question is under what conditions effort can be induced.

\subsubsection{The principal's problem}

The principal's strategy consists of a policy $\gamma(\mu):[0,1] \rightarrow[0,1]$ : given the fraction of projects that failed, she has to decide what fraction of the failures to punish $(\gamma)$. Assume that punishing at rate $\gamma \geq \gamma$ will induce the agent to exert effort (conditions for this to hold will be derived in 3.2.2). Since punishment is costly, the principal will in all cases choose either $\gamma=0$ or $\gamma=\chi$; anything between these two would be costly without inducing effort, while anything greater than $\underline{y}$ would be effective but unnecessarily costly. The question then becomes when the principal should punish and when she should not.

Given a policy $\gamma(\bar{f})$, let $\Gamma$ be the subset of the unit interval for which the principal punishes agents. That is,

$$
\Gamma \equiv\{\mu: \gamma(\mu)=\gamma\}
$$

For $\mu_{t} \in \Gamma$, agents know that they will be punished for failure with probability $\underline{\chi}$, and are thus induced to exert effort in these states.

If the principal never punishes $(\gamma(\mu)=0 \forall \mu)$, the agents will always shirk and the principal's 
expected value is simply the expected discounted value of production less wages:

$$
\underline{\mathrm{U}} \equiv \frac{1}{1-\delta}\{(1-\bar{\mu})+\bar{\mu} \pi(1+g)-w(1+\bar{\mu} g)\} .
$$

The principal's value of employing policy $\gamma(\cdot)$ given a technology state $\mu_{t}$ for which the principal does punish is

$$
\begin{gathered}
U^{P}\left(\gamma(\cdot), \mu_{t} \in \Gamma\right)=\left(1-\mu_{t}\right)+\mu_{t}[\pi(1-\varepsilon)(1+g)+\pi \varepsilon+(1-\pi)(1-\varepsilon)] \\
-(1-\pi) \varepsilon r\left(\mu_{t} \gamma\left(\mu_{t}\right)\right)-w\left(1+\mu_{t} g\right)+\delta \mathrm{E} U\left(\gamma(\cdot), \mu_{t+1}\right)
\end{gathered}
$$

The principal's value of employing policy $\gamma(\cdot)$ given a technology state $\mu_{t}$ for which the principal does not punish is

$$
U^{N}\left(\gamma(\cdot), \mu_{t} \notin \Gamma\right)=\left(1-\mu_{t}\right)+\mu_{t} \pi(1+g)-w\left(1+\mu_{t} g\right)+\delta \mathrm{E} U\left(\gamma(\cdot), \mu_{t+1}\right) .
$$

The difference is

$$
\mu_{t}[-\pi \varepsilon g+(1-\pi)(1-\varepsilon)]-(1-\pi) \varepsilon r\left(\gamma\left(\mu_{t}\right) \mu_{t}\right)
$$

For a policy $\gamma(\cdot)$ to be optimal, this must be greater than zero. Rearranging and using the fact that in equilibrium the principal will punish at rate $\underline{\gamma}$, we have

$$
\frac{(1-\pi)(1-\varepsilon)-\pi \varepsilon g}{(1-\pi) \varepsilon} \mu>r(\underline{\mu} \mu)
$$

This equation defines when the principal will prefer to punish if she is able to commit to a punishment policy $\gamma(\cdot)$. Figure 3 plots both sides of this equation for the sample parameters and functional forms given in Section 3.2.4. 
Definition 1. Let $\Gamma^{\star}$ be the set of all $\mu \in[0,1]$ that satisfy Equation 5-the set of $\mu$ for which the principal can credibly threaten punishment under commitment.

However, given that wages are realized before production, if the principal is unable to commit to a policy $\gamma(\cdot)$ then she must prefer punishing the agents to deviating to autarky for all $\mu_{t} \in \Gamma$ :

$$
-r\left(\underline{\gamma} \mu_{t}\right)+\delta \mathrm{E} U\left(\gamma(\cdot), \mu_{t+1}\right) \geq \delta \underline{\mathrm{U}} .
$$

The principal's expected utility is

$$
\begin{aligned}
\mathrm{E} U\left(\gamma(\cdot), \mu_{t}\right) & =\frac{1}{1-\delta} \int_{\mu \in \Gamma}\{\mu[-\pi \varepsilon g+(1-\pi)(1-\varepsilon)]-(1-\pi) \varepsilon r(\mu \chi)\} \mathrm{d} \mu \\
& +\frac{1}{1-\delta} \int_{0}^{1}\{(1-\mu)+\mu \pi(1+g)-w(1+\mu g)\} \mathrm{d} \mu .
\end{aligned}
$$

Definition 2. Let $\Gamma^{\star \star}$ be the set of all $\mu \in[0,1]$ that satisfy Equation 6-the set of $\mu$ for which the principal can credibly threaten punishment when commitment is not possible.

Proposition 1. Regardless of whether she can commit, the principal's optimal policy $\gamma(\mu)$ will be a threshold rule:

$$
\gamma^{\star}(\mu)= \begin{cases}\gamma & \mu \in[0, \tilde{\mu}) \\ \gamma \in\{0, \underline{\chi}\} & \mu=\tilde{\mu} \\ 0 & \mu \in(\tilde{\mu}, 1]\end{cases}
$$

(The optimal policy at exactly the threshold $\tilde{\mu}$ will depend on the distribution $Q(\mu)$ and the parameters of the game, and is explained in the proof.)

Proof. First consider the case with commitment. Since $r(\cdot)$ is convex, $r(\gamma \mu)$ is convex in $\mu$ given any $\gamma \in[0,1]$. Recalling that $r(0)=0$, this implies that given $\gamma$, if $\mu \in[0,1]$ satisfies Equation 5, then so does any $\mu^{\prime}$ satisfying $0<\mu^{\prime}<\mu$. So letting $\tilde{\mu}$ be the supremum of the set of values in $[0,1]$ for 
which Equation 5 holds, the principal can and will optimally punish for all $\mu$ up to the threshold $\tilde{\mu}$. As discussed before, the principal will in all cases choose either $\gamma=0$ or $\gamma=\gamma$. So under commitment the principal's optimal policy is the threshold rule given by Equation 7, with $\gamma^{\star}(\tilde{\mu})=\gamma$ if and only if $\tilde{\mu}$ satisfies Equation 5 .

Next, consider the case where the principal cannot commit to a policy $\gamma(\cdot)$. Consider a rule $\gamma(\mu):[0,1] \rightarrow\{0, \underline{\chi}\}$, and $\mu_{0}, \mu_{1} \in[0,1]$ such that $\mu_{0}<\mu_{1}$. Since $r(\cdot)$ is increasing, if $\mu_{1}$ satisfies Equation 6 then so does $\mu_{0}$. So if $\gamma\left(\mu_{1}\right)=\chi, \gamma\left(\mu_{0}\right)=\chi$. Letting $\tilde{\mu}$ be the supremum in $[0,1]$ such that Equation 6 holds, the principal will again follow a threshold rule given by Equation 7, with $\gamma^{\star}(\tilde{\mu})=\chi$ if and only if $\tilde{\mu}$ satisfies Equation 6 .

If $r(\cdot)$ is differentiable at zero, a sufficient condition for $\Gamma^{\star}$ to be non-empty is

$$
\frac{(1-\pi)(1-\varepsilon)-\pi \varepsilon g}{(1-\pi) \varepsilon r^{\prime}(0)}>\chi
$$

Otherwise, Equation 5 would hold with equality only at $\mu=0$, after which the costs of punishment would outweight the benefits. While the case without commitment is perhaps more realistic, this paper will focus on the case with commitment, to emphasize that the driving friction here is not necessarily a commitment problem but rather the convexity of the principal's punishment function.

\subsubsection{The agent's problem}

Assume the chance of punishment is $\gamma(\mu)$ for agents who fail to produce, which may depend on the technology state $\mu$, as this governs the maximum number of agents whose production can fail in the period. The agent will exert effort if the expected value of doing so exceeds the expected value of shirking. The value function therefore depends on the usefulness of this period's new technology, $\mu_{t}$. The agent is guaranteed $w a_{i t}$ and pays $c$ if the technology is useful to him (chance $\mu_{t}$ ), and continues in the game $\left(\mathrm{EV}\left(\mu_{t+1}\right)\right)$ if the new technology is good (chance $\left.\pi\right)$ and the agent receives the correct signal 
(chance $1-\varepsilon$ ). If either the technology is good but the signal wrong (chance $\pi \varepsilon$ ) or the technology is bad and the signal correct (chance $(1-\pi)(1-\varepsilon)$ ), the agent forgoes using the new technology and continues on in the game $\mathrm{EV}\left(\mu_{t+1}\right)$. Only if the agent receives a false positive signal (chance $(1-\pi) \varepsilon)$ is punishment triggered, and even then there is only a $\gamma(\mu)$ chance of receiving it, with the agent continuing on $\left(\mathrm{EV}\left(\mu_{t+1}\right)\right)$ with complementary probability. If the technology is not useful to the agent (chance $\left.1-\mu_{t}\right)$, the agent continues on in the game with $\mathrm{E} V\left(\mu_{t+1}\right)$.

$$
\begin{gathered}
\mathrm{E} V\left(\mu_{t}\right)=w+\int_{\mu \in \Gamma} \mu\left\{\pi\left[(1-\varepsilon)\left(w g+\delta \mathrm{E} V\left(\mu_{t+1}\right)\right)+\delta \varepsilon \mathrm{E} V\left(\mu_{t+1}\right)\right]\right. \\
\left.+\delta(1-\pi)\left[(1-\varepsilon) \mathrm{E} V\left(\mu_{t+1}\right)+\varepsilon\left(\gamma\left(\mu_{t}\right) b+\left(1-\gamma\left(\mu_{t}\right)\right) \mathrm{E} V\left(\mu_{t+1}\right)\right)\right]\right\} \mathrm{d} Q(\mu) \\
+\int_{\mu \notin \Gamma} \mu\left\{\delta \mathrm{E} V\left(\mu_{t+1}\right)+\pi w g\right\} \mathrm{d} Q(\mu)+\delta \int_{0}^{1}(1-\mu) \mathrm{E} V\left(\mu_{t+1}\right) \mathrm{d} Q(\mu) .
\end{gathered}
$$

Simplifying and gathering terms, this becomes

$$
\begin{gathered}
\mathrm{E} V\left(\mu_{t}\right)=w+\int_{\mu \in \Gamma} \mu[\pi(1-\varepsilon) w g+\delta(1-\pi) \varepsilon \gamma(\mu) b] \mathrm{d} Q(\mu)+\int_{\mu \notin \Gamma} \mu \pi w g \mathrm{~d} Q(\mu) \\
+\delta\left\{\int_{\mu \in \Gamma} \mu[\pi+(1-\pi)(1-\varepsilon \gamma(\mu))] \mathrm{d} Q(\mu)+\int_{\mu \notin \Gamma} \mu \mathrm{d} Q(\mu)+\int(1-\mu) \mathrm{d} Q(\mu)\right\} \mathrm{E} V\left(\mu_{t+1}\right) .
\end{gathered}
$$

Using the fact that $\mathrm{E} V\left(\mu_{t+1}\right)=\mathrm{E} V\left(\mu_{t}\right)$ and solving for $\mathrm{E} V\left(\mu_{t}\right)$ yields

$$
\mathrm{E} V\left(\mu_{t}\right)=\frac{w+\int_{\mu \in \Gamma} \mu[\pi(1-\varepsilon) w g+\delta(1-\pi) \varepsilon \gamma(\mu) b] \mathrm{d} Q(\mu)+\int_{\mu \notin \Gamma} \mu \pi w g \mathrm{~d} Q(\mu)}{1-\delta\left\{\int_{\mu \in \Gamma} \mu[\pi+(1-\pi)(1-\varepsilon \gamma(\mu))] \mathrm{d} Q(\mu)+\int_{\mu \notin \Gamma} \mu \mathrm{d} Q(\mu)+\int(1-\mu) \mathrm{d} Q(\mu)\right\}}
$$

For an agent for whom $\mu_{i t}=1$ when $\mu_{t} \in \Gamma$, with $\gamma\left(\mu_{t}\right)=\gamma$, the expected value of effort is 
$w-c+\pi(1-\varepsilon)\left(w g+\delta \mathrm{E} V\left(\mu_{t+1}\right)\right)+\delta(\pi \varepsilon+(1-\pi)(1-\varepsilon)) \mathrm{E} V\left(\mu_{t+1}\right)+\delta(1-\pi) \varepsilon\left(\gamma b+(1-\gamma) \mathrm{E} V\left(\mu_{t+1}\right)\right)$.

The expected value of shirking is

$$
w+\pi\left(w g+\delta \mathrm{E} V\left(\mu_{t+1}\right)\right)+\delta(1-\pi)(1-\gamma) \mathrm{E} V\left(\mu_{t+1}\right)+\delta(1-\pi) \gamma b
$$

The difference is

$$
-c-\pi \varepsilon w g+\delta(1-\pi)(1-\varepsilon) \gamma\left[\mathrm{E} V\left(\mu_{t+1}\right)-b\right] .
$$

So the agent will exert effort if the increased chance of continuation in the game compared with the outside option outweighs cost of effort and loss owing to false negative signals:

$$
\delta(1-\pi)(1-\varepsilon) \gamma\left[\mathrm{E} V\left(\mu_{t+1}\right)-b\right]>c+\pi \varepsilon w g
$$

Equation 8 can be used to yield a condition for effort containing only parameters. So given a policy of the principal $\gamma(\mu)$, Equation 9 determines when the agents will exert effort. Figure 2 plots both sides of Equation 9 for the sample functional forms and parameters given in Section 3.2.4.

Definition 3. Let $\chi$ be the lowest possible punishment rate which induces effort (Equation 9 holds). With smooth expected values this is

$$
\chi:=\frac{c+\pi \varepsilon g \mathrm{E} V\left(\mu_{t+1}\right)}{\delta(1-\pi)(1-\varepsilon)\left[\mathrm{E} V\left(\mu_{t+1}\right)-b\right]}
$$

It is obvious from Equation 9 that if costs and the outside option are positive, the value of the signal alone is not enough to induce effort in the absence of punishment $(\gamma=0)$. That is, only the threat of punishment can induce effort. To further ensure this is an interesting problem, assume that the level 
of noise $\varepsilon$ is small enough that if failure is always punished $(\gamma=1)$, effort is induced. Equivalently, assume that

$$
\chi \in[0,1]
$$

In other words, assume that the noisiness of the signal is not so high as to prevent the principal from being able to induce effort. An equilibrium with effort is then simply characterized by $\{\gamma, \tilde{\mu}\}$, where $\tilde{\mu}$ characterizes the principal's policy rule $\gamma(\mu)$ of the form given by Equation 7, and $\gamma$ is characterized by Equation 9. More specific characterization requires functional forms, as in the example (Section 3.2.4).

\subsubsection{Welfare loss}

The equilibrium with effort only differs from the first-best case when $\mu_{t}>\tilde{\mu}$. In such a state, expected aggregate output is

$$
\int_{0}^{1} f_{i t} \mathrm{~d} i=\left(1-\mu_{t}\right)+\mu_{t} \pi(1+g)
$$

The expected welfare loss for $\mu_{t}>\tilde{\mu}$ (compared to the first best, Equation 4) is thus

$$
\mu_{t}[(1-\varepsilon)(1-\pi)-\pi g \varepsilon+c]
$$

If Equations 2 and 3 hold this welfare loss will be positive. 


\subsubsection{Example}

To aid understanding, this section considers a case with simple functional forms and some sample parameters. Let the principal's cost of punishing $\mu$ agents be

$$
r(\mu)=R \mu^{2}
$$

Then from Equation 5, the principal's condition for punishment (assuming she can commit to a policy) becomes

$$
\frac{(1-\pi)(1-\varepsilon)-\pi \varepsilon g}{(1-\pi) \varepsilon} \mu>R \chi^{2} \mu^{2}
$$

The highest measure of failures the principal is willing to punish is then

$$
\tilde{\mu}=\frac{(1-\pi)(1-\varepsilon)-\pi \varepsilon g}{(1-\pi) \varepsilon R \gamma^{2}}
$$

Intuitively, as the rate of punishment rises, the largest group the principal is willing to punish shrinks.

Assume a uniform distribution for $\mu$ on $[0,1]$, such that

$$
Q(\mu)= \begin{cases}0 & \mu<0 \\ \mu & \mu \in[0,1] . \\ 1 & \mu>1\end{cases}
$$

Given that the principal employs a threshold strategy (equation 7), the agent's expected continuation value $\mathrm{EV}\left(\mu_{t}\right)$ (Equation 8$)$ becomes

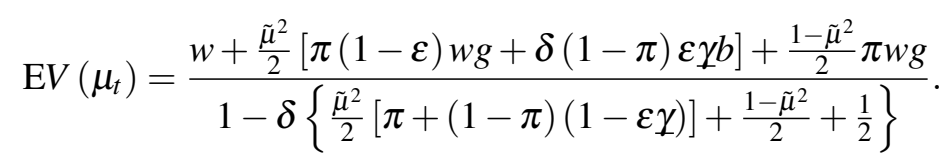




\subsubsection{No commitment}

Since the principal will use a threshold strategy in equilibrium, her expected utility in equilibrium will be

$$
\begin{gathered}
\mathrm{E} U\left(\gamma^{\star}(\cdot), \mu_{t}\right)=\tilde{\mu}\left\{\left(1-\frac{\tilde{\mu}}{2}\right)+\frac{\tilde{\mu}}{2}[\pi(1-\varepsilon)(1+g)+\pi \varepsilon+(1-\pi)(1-\varepsilon)]\right. \\
\left.-(1-\pi) \varepsilon R \frac{\tilde{\mu}}{2} \chi^{2}-w\left(1+\mu_{t} g\right)\right\} \\
+(1-Q(\tilde{\mu}))\left\{\left(1-\mu_{t}\right)+\mu_{t} \pi(1+g)-w(1+\bar{\mu} g)\right\}+\delta \mathrm{E} U\left(\gamma(\cdot), \mu_{t+1}\right) .
\end{gathered}
$$

This can be simplified to

$$
\begin{gathered}
\mathrm{E} U\left(\gamma^{\star}(\cdot), \mu_{t}\right)=\int_{\mu \in \Gamma}\{\mu[-\pi \varepsilon g+(1-\pi)(1-\varepsilon)]-(1-\pi) \varepsilon r(\mu \chi)\} \mathrm{d} \mu \\
+\int_{0}^{1}\{(1-\mu)+\mu \pi(1+g)-w(1+\mu g)\} \mathrm{d} \mu+\delta \mathrm{E} U\left(\gamma(\cdot), \mu_{t+1}\right) .
\end{gathered}
$$

\subsubsection{Sample parameters and results}

Consider the sample parameters in Table 1, for which Equations 2 and 3 both hold. 
Table 1: Example parameter values

\begin{tabular}{crl} 
Parameter & Value & Description \\
\hline \hline$\delta$ & 0.95 & Common discount rate \\
$\pi$ & 0.85 & Chance that new technology is sound \\
$\varepsilon$ & 0.01 & Chance that signal is wrong \\
$b$ & 0 & Agent's outside option \\
$w$ & 0.20 & Agent's share of potential productivity \\
$g$ & 0.25 & Growth rate \\
$c$ & 0.03 & Agent's cost of research effort \\
$R$ & $1 E 6$ & Principal's punishment cost parameter \\
\hline
\end{tabular}

Given these parameters, Equation 12 gives the maximum measure of agents that the principal is willing to punish in equilibrium; Figure 1 plots $\tilde{\mu}$. 
Figure 1: The principal's willingness to punish as a function of punishment rate

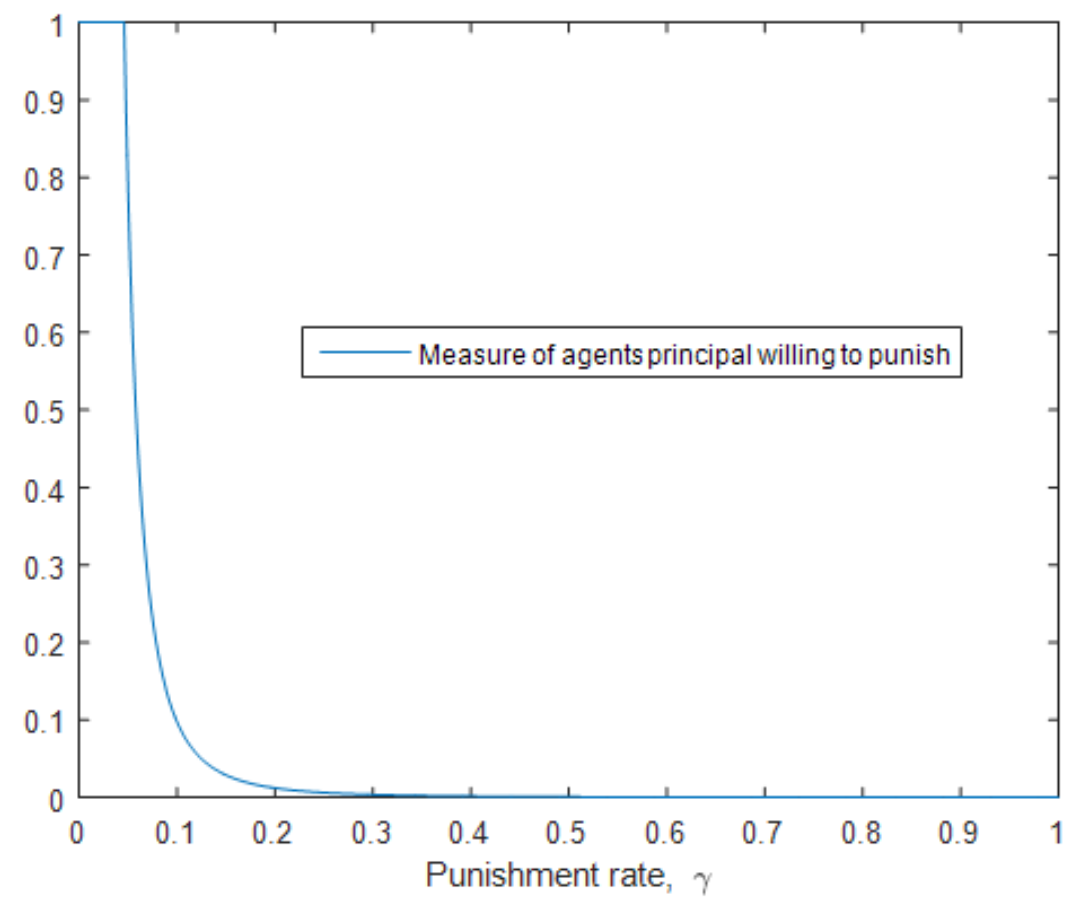

As expected, the willingness to punish falls with the punishment rate. Given this willingness to punish, the agent's costs of effort (right hand side of Equation 9) and benefits (left hand side) are given by Figure 2 as a function of punishment rate $\gamma$. The agent is only willing to exert effort if threatened with a punishment rate of $\gamma \geq 5 \%$ - this is where benefits outstrip costs. ${ }^{6}$

${ }^{6}$ For the case without commitment, the lower bar is roughly double $(10.8 \%)$. 
Figure 2: The agent's tradeoff as a function of punishment rate

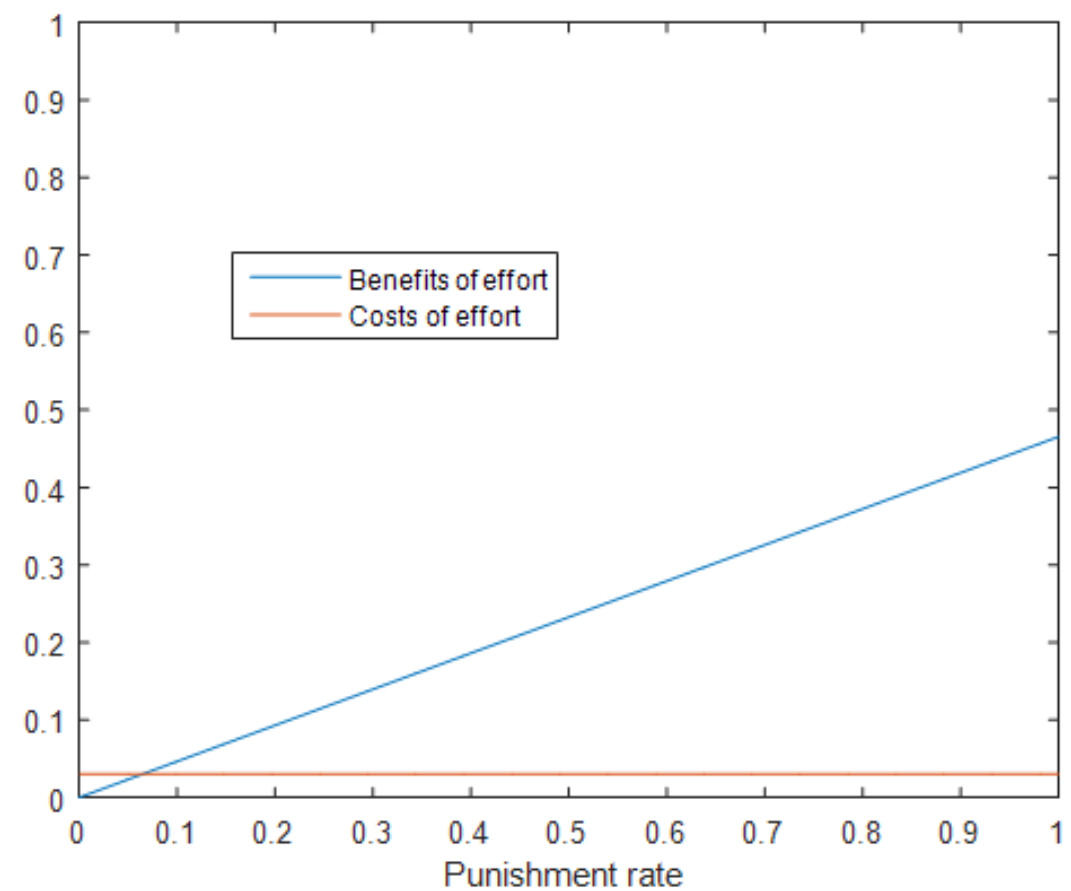

Finally, given $\chi=5 \%$, Figure 3 plots the principal's costs (right hand side of 5) against her benefits (left hand side). She is willing to punish a group of agents as large as 0.91 , but no larger. This demonstrates the main mechanism of the paper: the principal may be unwilling to punish large groups of shirkers. 
Figure 3: The principal's tradeoff in equilibrium

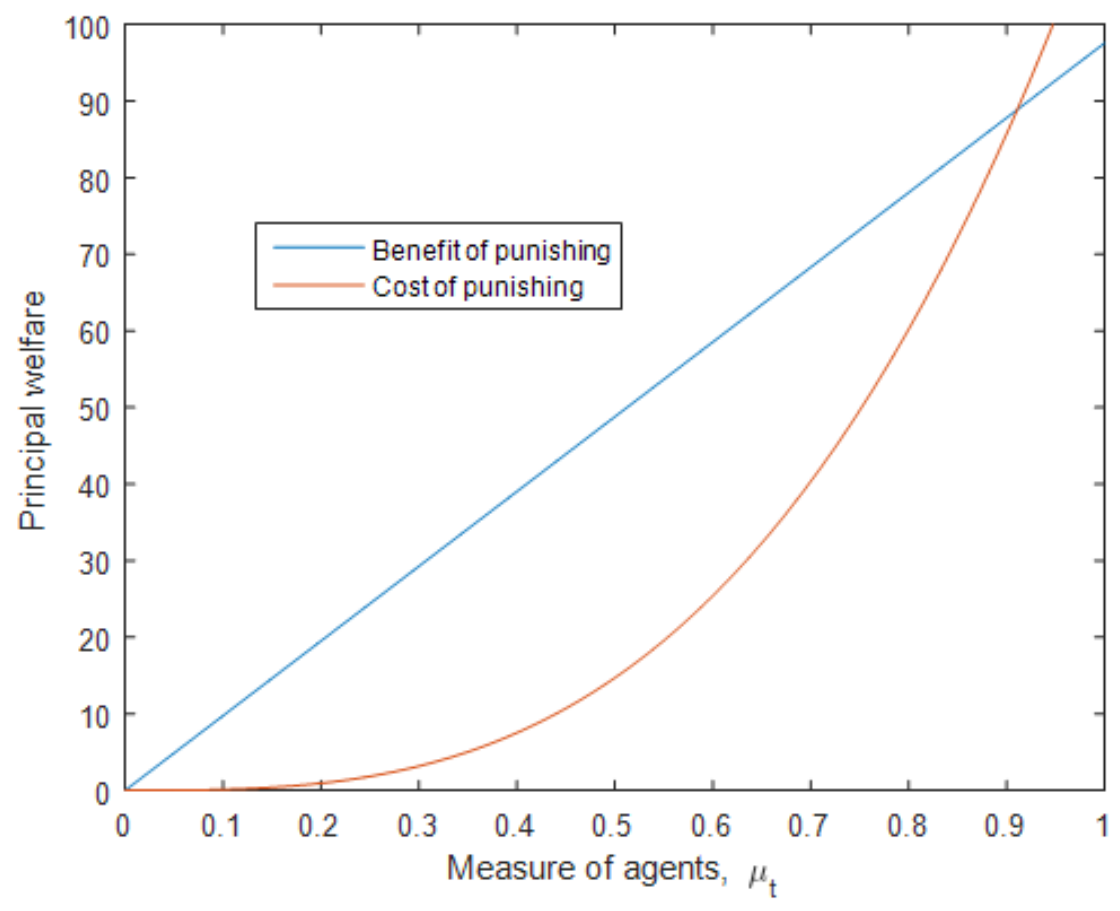

In this equilibrium, workers shirk whenever $\mu>\tilde{\mu}=0.91$, since they know they will not be punished. Figure 4 shows expected one-period welfare in this equilibrium compared to the firstbest case with full effort and the autarkic equilibrium with no punishment. Total expected welfare (integrating over $\mu$ ) is greater in the equilibrium with effort, but falls well short of the first best. Furthermore, in equilibrium both production and welfare exhibit nonmonotonicity in the applicability of technology $\mu$, as technology that is useful to everyone facilitates coordinated shirking. 
Figure 4: Total welfare

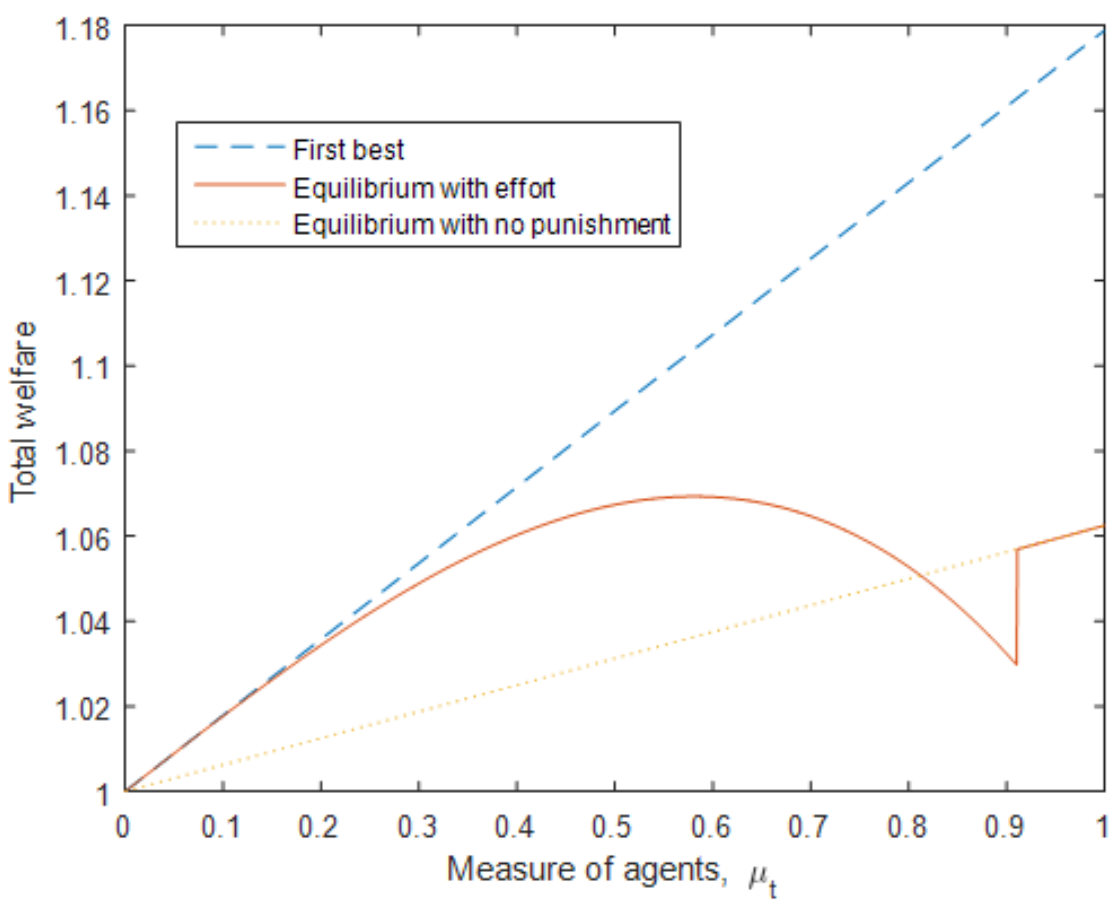

\section{Applications and discussion}

\subsection{Firms, workers, and aggregate downturns}

Applied to a worker-firm setting, this model has a surprising prediction: a technological advance that is useful to too many people may actually trigger a downturn in production. This is the main result of the paper. Consider a new technology which is useful to many people and will work well most of the time, represented in the model by a string of realizations of $\mu$ which are all higher than $\tilde{\mu}$ and a string of realizations of $\chi$ which are mostly one. 
Table 2: A (potentially) useful technology

\begin{tabular}{c|cccc}
$t$ & 1 & 2 & 3 & 4 \\
\hline \hline$\mu_{t}$ & $>\tilde{\mu}$ & $>\tilde{\mu}$ & $>\tilde{\mu}$ & $>\tilde{\mu}$ \\
$\chi_{t}$ & 1 & 1 & 1 & 0 \\
\hline
\end{tabular}

Since $\mu_{t}>\tilde{\mu}$, which corresponds to a technological innovation relevant to a large fraction of workers, the firm is unable to credibly threaten to fire workers who fail to produce because the cost of replacing them is too high. So in these states, workers know they can shirk with impunity, as the firm can't fire everyone. Production in this example will be high for the first three periods but fail in the fourth for the large fraction of workers who are using the new technology. It is important to note that such states (with $\mu_{t}>\tilde{\mu}$ ) may arise rarely. The firm may in fact think that it is not subject to such agency frictions simply because a useful enough technology (high enough $\mu_{t}$ ) has not come along in a while. The model can thus link the failure of models used to price securitized products to their popularity.

Furthermore, consider a worker who somehow learns that the new, widely useful technology is flawed $\left(\chi_{t}=0\right)$. Even with this knowledge, he will still choose to use the new technology if everyone else is, as it nets him a higher wage, and there is no chance of punishment. Research effort in this model has the flavor of a public good, since the technology is common. If it were possible for just one worker to exert the research effort and then share the signal with the others before technology choices were made, that would fix the problem. But once everyone is using a technology, it may be difficult to dissuade them, even if its flaws are made known. This may explain why even as doubts regarding the soundness of the credit boom arose, industry practices were slow to change.

Of course, many financial services employees were indeed fired or laid off during the financial crisis. This paper's model predicts total amnesty for large enough groups of shirkers, but it is simplistic in that the firm size remains the same — if the firm size shrank during recessions, for example, there 
could be layoffs during a downturn. And since workers would not individually be able to affect the probability of a downturn, the core mechanism of the model would remain intact. Also, the model can arguably be applied at an industry level rather than just a firm level. A quant using industry-standard technology could reasonably expect this choice not to affect his future employability, even if said technology turned out to be unsound.

\subsection{Policy implications}

Following the financial crisis of 2008, capping bankers' bonuses was widely discussed as a means of controlling risk-taking. In 2013, the European Union restricted banker bonuses to $100 \%$ of pay (or up to $200 \%$ with shareholder approval).(PricewaterhouseCoopers LLP 2013) This shifts the balance of bankers' compensation from variable (bonus) to fixed (salary). Can such a policy reduce risk-taking?

In this paper, the vital friction arises precisely because firms cannot punish workers by paying them less, only by firing them. Restricting variable compensation may thus exacerbate this friction, and increase the chance of a downturn. Variable compensation aligns workers' incentives with the returns of their projects. If in the model firms were allowed to pay workers their realized production rather than their potential production (similar to end-of-year bonuses), workers would be motivated to put forth research effort all the time, and the friction would disappear.

Of course, timing is key. If the outcomes of workers' projects are not realized for years after they are paid, then end-of-year variable compensation would be no different from fixed compensation during the year. In this case, something stronger would be needed, such as clawbacks (in which the firm is able to reclaim bonuses if long-term performance objectives are not met) or payment in stock or stock options (which would lose value if the projects failed). These prescriptions are not new, but this paper illuminates another reason they may be important: the inadequacy of firing workers as a punishment to induce effort.

Finally, the example of speeding (discussed in the Appendix) suggests another possible solution. If 
firms are able to somehow identify a salient individual for any possible subset of workers and commit to punishing that individual, coordinated shirking can be avoided. For example, the firm could commit to firing the most senior worker (in terms of tenure at the firm) in a group whenever their production fails. Of course, this may not always be possible, or enough. Workers may know that a lot of other people are using a new technology but remain unsure exactly who. Or a group of workers may have all started at the same time, making seniority an insufficiently distinguishing characteristic. Nonetheless, to the extent that firms can create a complete, strict ordering of workers that is known by all, it may be able to prevent instances of coordinated shirking.

\section{References}

Acharya, V. V. and Richardson, M. (2009). Causes of the financial crisis. Critical Review, 21(23):195-210.

Acharya, V. V. and Yorulmazer, T. (2007). Too many to fail — An analysis of time-inconsistency in bank closure policies. Journal of Financial Intermediation, 16(1):1-31.

Acharya, V. V. and Yorulmazer, T. (2008). Cash-in-the-Market Pricing and Optimal Resolution of Bank Failures. Review of Financial Studies, 21(6):2705-2742.

Becker, G. S. (1968). Crime and Punishment: An Economic Approach. Journal of Political Economy, 76(2):169.

Coval, J., Jurek, J., and Stafford, E. (2009). The Economics of Structured Finance. Journal of Economic Perspectives, 23(1):3-25.

Devenow, A. and Welch, I. (1996). Rational herding in financial economics. European Economic Review, 40(3-5):603-615. 
DiPasquale, D. and Glaeser, E. L. (1998). The Los Angeles Riot and the Economics of Urban Unrest. Journal of Urban Economics, 43(1):52-78.

Farhi, E. and Tirole, J. (2012). Collective Moral Hazard, Maturity Mismatch, and Systemic Bailouts. American Economic Review, 102(1):60-93.

Hall, R. E. (2010). Why Does the Economy Fall to Pieces after a Financial Crisis? The Journal of Economic Perspectives, 24(4):3-20.

MacKenzie, D. and Spears, T. (2014). 'The Formula that Killed Wall Street': The Gaussian Copula and Modelling Practices in Investment Banking. Social Studies of Science, 44(3):393-417.

PricewaterhouseCoopers LLP (2013). EU Bonus Cap Update - Cap to Apply More Broadly. FS Regulatory Brief.

Rajan, R. (2008). Bankers' pay is deeply flawed. Financial Times, 8.

Taylor, J. B. (2009). The Financial Crisis and the Policy Responses: An Empirical Analysis of What Went Wrong. Working Paper 14631, National Bureau of Economic Research.

Tokyo, M. N. i. and York, D. W. i. N. (2007). Citigroup chief stays bullish on buy-out. Financial Times (FT.Com).

Zimmer, D. M. (2012). The Role of Copulas in the Housing Crisis. Review of Economics and Statistics, 94(2):607-620.

\section{Appendix: Herding in Crime}

This model can be used to explain herding behavior in crime, such as looting, or speeding on the highway. Consider the latter example, with the agents cast as motorists and the principal in the role 
of highway patrol officer. Let $\mu_{t}$ represent the number of drivers who, by chance, choose to traverse a certain stretch of highway in period $t$. Those drivers travelling the highway (those for whom $\mu_{i t}=1$ ) can either drive slowly (effort) or quickly (shirk). Driving slowly is more costly $(c)$ as it takes longer, but allows a driver to see (most of the time, $1-\varepsilon$ percent) whether the patrol officer is there $\left(\chi_{t}=0\right)$ or not $\left(\chi_{t}=1\right)$. If the patrol officer is there, she'd prefer to punish the driver. The officer may have an extremely convex punishment function $r(\cdot)$, since she may only be able to pull over a fixed number of drivers per period, $\tilde{\mu}$. So a driver may obey the speed limit unless he finds himself driving with a large pack of other drivers $(\mu>\tilde{\mu})$, at which point the chance of getting pulled over decreases and he may choose to speed.

In this application, it is perhaps unrealistic to assume that the patrol officer will not punish any drivers when they are numerous $(\mu>\tilde{\mu})$. Because it is her job (or more cynically, to fulfill a quota), she may still pull over as many speeding drivers as she can. Note, however, that as long as she pulls over speeders randomly, this will not induce any change of behavior on the part of the drivers, since any one driver's expected chance of punishment will be less than $\chi$ when $\mu>\tilde{\mu}$.

\section{Policy implications}

Speeding drivers have an important characteristic not included in this paper's model: they are ordered. And crucially, if the first driver in a pack of speeders slows down, he ceases to be first. So the patrol officer only needs to commit to pulling over the first car in any speeding pack; this will motivate the first driver to slow down until he is no longer the first. Then the second driver will become the first and do the same, and so on, until no one is speeding.

This example highlights how coordinated shirking can be avoided if given any set of shirkers, one is somehow salient. Even when the principal is unable to credibly punish a large group, she can credibly threaten to always punish the salient individual. As long as everyone knows who the salient individual is for any possible subset of agents and the principal is able to condition punishment 
on the characteristic that distinguishes him, this will unravel groups of shirkers and allow for a fulleffort equilibrium. The easiest way to do this is one that arises naturally in the speeding example: a complete, strict order over all agents. ${ }^{7}$

\footnotetext{
${ }^{7}$ Technically, all that is needed is a map $f(\cdot)$ from the power set of agents to agents such that $f(a) \in a$; a complete strict order is simply an easy example.
} 